\title{
PER UN CONTRIBUTO AL DIALETTO ISTRIOTO DI ROVIGNO: OSSERVAZIONI SUL SAGGIO DI DIALETTO ROVIGNESE - IL MENDICANTE D'AMORE DI ANDRJA UORGANI (A CURA DI PIETRO KANDLER)
}

Abstract: Nel lavoro si presenta un'analisi stilistica e metrica comparata tra un poemetto in dialetto istrioto di Rovigno, pubblicato nel 1846, e testi di poeti della tradizione classica italiana del Quattrocento. Dal raffronto emergono stilemi e motivi tipici della poesia aulica, quali l'amore non corrisposto, la sofferenza da ciò causata, la derisione dell'innamorato da parte dell'aggraziata giovane, ma anche il tono ironico - e appena celato - dell'autore, il tutto però elaborato in chiave popolareggiante attraverso l'accorto uso del dialetto istrioto di Rovigno'.

Parole chiave: Rovigno, dialetto, istrioto, poemetto, Kandler, Istria.

Circa vent'anni dopo le prime testimonianze in dialetto istrioto di Dignano d'Istria - apparse su fogli volanti tra il secondo e il terzo decennio del XIX secolo -, assistiamo, nel 1846, alla pubblicazione sulla rivista 'L'Istria" di Trieste, diretta da Pietro Kandler ${ }^{2}$, di un poemetto in ottava rima

*scergna@unipu.hr

${ }^{1}$ Rovigno (in croato Rovinj) è una città di circa 13000 abitanti, situata sulla costa occidentale dell'Istria. Seguì, fino alla fine della Seconda guerra mondiale, le alterne vicende storiche dell'Italia nordorientale, per passare, nel 1947, sotto la giurisdizione jugoslava e, dal 1992, sotto quella croata. Vi si conserva ancora, dai pochi parlanti italiani rimasti in seguito all'esodo del 1945, l'antico dialetto istrioto nella variante rovignese. Tale idioma neolatino, preveneto, era parlato fino al XIII-XIV secolo in gran parte dell'Istria sudoccidentale, con propaggini anche a nord e all'interno della penisola; sopravvive ancora profondamente venetizzato - nei paesi di Valle, Gallesano e Sissano. A Fasana si è estinto intorno alla seconda metà del XX secolo; a Dignano non si parla più da una decina di anni circa. Cfr. Tekavčić (1980), Ursini (1989), Cergna (2012: 3-15), Crevatin (2013: 9-11).

${ }^{2}$ Sulla nascita della rivista triestina e sulla ricca e proficua attività storiografica svolta dal Kandler assieme ai suoi collaboratori, cfr. Budicin (2015). Per ulteriori fonti sul 
dal titolo Saggio di dialetto Rovignese. Il poemetto però - forse per motivi di spazio -, non fu pubblicato interamente sulle pagine della rivista, che si limitò a riportare quattordici ottave inframmezzate da quattro indicazioni sulla relativa omissione del testo, per cui - ad oggi - ci è ignota la versione originaria e completa del componimento rovignese. Né la breve nota posta in calce al testo stesso fornisce notizie più approfondite a proposito del poemetto. Scrive, infatti, il Kandler: "Questi brani di poesia sono tolti da un poemetto inedito scritto nel 1843, in cui l'Autore fa parlare uno stupido mendicante, figurandolo un poeta estemporaneo"s.

Dai versi, però, emerge il nome del poeta estemporaneo: Andrja Uorgani, verosimilmente un nome fittizio dietro cui forse si cela l'autore reale della poesia, o semplicemente un nome d'invenzione con il quale l'autore ha voluto indicare il protagonista del poemetto. Ma un altro nome ancora, che compare nel precedente n. 16-17 de "L'Istria" dobbiamo qui prendere in considerazione: Nino Vasgabrina (probabilmente trattasi anche qui di pseudonimo) il quale, il 15 marzo 1846, inviò alla redazione "la traduzione delle due solite narrazioncelle in dialetto rovignese" 4 perché, come apprendiamo dalle parole del redattore della rivista, non riteneva che "quelle pubblicate nel numero precedente" rispecchiassero la genuina espressione vernacola, ma "si scostassero dal vero" a causa dell'errata trascrizione della pronuncia originaria ${ }^{6}$. Possiamo fin d'ora constatare, quindi, l'interesse che fin dall'inizio della pubblicazione de "L'Istria" il Kandler dedicò, accanto ai suoi principali interessi storico-archeologici, alla produzione letteraria, e poetica in particolare, nei vari dialetti della penisola. Interesse che permarrà, come attestano le pagine della rivista, fino allo spegnimento del foglio, nel 1852.

Nel numero 13-14 la rivista pubblicava il primo di una serie di articoli dal titolo Saggio di dialetti istriani in cui trovarono spazio soprattutto trascrizioni di "narrazioncelle" - come le due di cui sopra-, dialoghi, componimenti poetici, brani tratti da antichi statuti e riportati, di volta in volta, in uno dei dialetti dell'Istria, nonché corredati da note esplicative con rispettiva introduzione (tra quelli considerati, troviamo a più riprese pubblicazioni in rovignese e dignanese, le uniche di area istriota). Nel numero succitato venivano presentate

settimanale kandleriano e sulla eterogeneità storiografica dei contributi, si veda ancora: Salimbeni (1991), Radossi (2014).

${ }^{3}$ In "L'Istria", a. I, 30 maggio 1846, n. 31-32, p. 128.

${ }^{4}$ In "L'Istria", a. I, 28 marzo 1846, n. 16-17, p. 61. Si tratta di due brevi favole gnomico-morali. Si veda anche, a p. 47 in nota, la lettera del sette giugno 1846 del dalla Zonca al Kandler, sulle "due narrazioncelle".

${ }^{5}$ Ibid. Per "numero precedente" il Kandler intendeva il numero 13-14, pubblicato il 14 marzo 1846.

${ }^{6}$ Ibid. 
due storielle nei dialetti di Trieste, Rovigno e Dignano ${ }^{7}$ : quella di due uomini e di una mannaia, e un'altra, più precisamente una favola, in cui si narra della formica operosa e previdente e della cicala spensierata e perdigiorno. Il Vasgabrina quindi, non soddisfatto della versione rovignese dei due brevi scritti, si premurò di far pervenire al giornale una diversa stesura degli stessi, che la redazione pubblicò nel n. 16-17 con alcune brevi note introduttive nelle quali il redattore, rivolgendosi a "Persona, che assunse nome (come sembra) immaginario", lo ringraziava del favore e lo pregava di inviargli "qualche saggio di più lungo discorso" ". La conferma che il Vasgabrina non sia rimasto sordo all'invito rivoltogli dal Kandler, è data dalla risposta di quest'ultimo al rovignese, in cui lo informa che

La Redazione dell'“Istria" ha ricevuto i versi inviati col foglio dei primi d'aprile corr., ne rende grazie, e si chiama avventurata di trovarsi in contatto con sì operoso e zelante cultore di uno dei dialetti istriani. La Redazione ha d'uopo di venire a più vicino concambio di pensieri e parole. Se la persona che assunse il nome di Nino Vasgabrina dubita che il Redattore sappia custodire un segreto, o se speciali motivi gli impediscano di manifestarsi, noi lo rispetteremo; solo ricerchiamo d'indicarci il modo con cui sia possibile di fargli pervenire nostre lettere. ${ }^{10}$

Da quanto finora riportato risulta che dal n. 19-20 fino al n. 31-32 sulle pagine de "L'Istria" non compare pubblicato alcun componimento in versi in dialetto rovignese, come pure in nessun altro vernacolo istrioto. Sarà solo in quest'ultimo numero, datato 30 maggio $1846^{11}$, che il Kandler pubblicherà con il titolo di Saggio di dialetto Rovignese il lamento d'amore inviatogli, è ipotizzabile, da tale Nino Vasgabrina, dietro il cui pseudonimo potrebbe forse celarsi Piero Angelini, autore del poemetto Lementi de Fimjta incontro Pjiro su murùs, o altri appartenenti alla famiglia Angelini ${ }^{12}$. In calce

${ }^{7}$ Le trascrizioni in dialetto istrioto di Dignano sono di Giovanni Andrea dalla Zonca.

8 “L'Istria", n. 16-17, cit.

${ }^{9}$ Ibid.

${ }^{10}$ In "L'Istria”, a. I, 11 aprile 1846, n. 19-20, p. 73. Purtroppo, dalle ricerche effettuate presso l'Archivio diplomatico della Biblioteca civica di Trieste, non è emersa la lettera cui fa riferimento il Kandler nella sua risposta al Vasgabrina.

${ }^{11}$ Da questa fonte citeremo d'ora in poi i versi tratti dal Saggio, pubblicato alle pagine 127 e 128.

12 È qui importante notare come non pochi fossero i membri dell'illustre famiglia rovignese degli Angelini, soprattutto nel XVIII e XIX secolo (e fino ad oggi con il fine poeta in istrioto rovignese, Gianclaudio Angelini), a dedicarsi alla composizione poetica. Così, da una ricerca di Giovanni Radossi e Antonio Pauletich (1976: 247-277), veniamo a sapere che "Antonio Angelini fu Angelo (morto nel 1808) fu egregio cultore delle Muse e dotto giureconsulto. [...] Pubblicò parecchie composizioni poetiche volanti, od inserite in raccolte del momento" (F. Glezer, Memorie di Rovigno, Bontempo, Pola 1885, in G. 
al componimento, accanto alla breve postilla di cui sopra, sono riportate delle essenziali note esplicative nelle quali s'informa il lettore sulla giusta pronuncia dei dittonghi rovignesi, sulle soluzioni adottate dalla redazione nella trascrizione dei versi, nonché sul significato di alcuni termini di più difficile interpretazione. Nessun riferimento però viene fatto all'autore del testo, in osservanza, evidentemente, con quanto espresso dal Vasgabrina nella lettera "dei primi d'aprile" (fig. 1).

canto dell' inno della 'Vergine, cui l' eco delle colline ripetendo moltiplica, più grata rendendone l'armonia, e fra lo sparo d'armi d'ogni maniera intrecciando carole la festeggiano. T' imagina di vedere una madre círcondata da' suoi figliuoletti che pieni di esultanza le saltellano intorno, e chi una mano le prende e chi l'altra, affettuosamente baciandola; quale le si attacca alla gonna, quale le ginocchia-le abbraccia, sponendo le cento cose in un multisono cicalio, ed ora da una parte, ora dall' altra le fanno amoroso assalto per avere un bacio, una carezza. Durante il tragitto, che all' incirca è di mezz' ora, parte della gente di processione, e i cavalli girano il seno alla spicciolata, per andarsi a ricongiungere a' suoi presso la chiesetta di $\boldsymbol{S}$. Felice, e la musica avviva delle sue armonie l' allegrezza della toccante cerimonia.

Chi pensasse essere codesto uno spettacolo da profanare la sacra funzione, piuttostochè infervorare gli animi nella divozione, consideri le esteriori formalità e le pompe del culto religioso; consideri, e quì gridi pure, que'canti e quelle musiche che fra le sesse pareti del santuario costringono quasi gli animi più bisognosi di esterni eccitamenti a raccogliersi nel sentimento della pietà e della fede, a pensare invece agli amori del $\mathbf{P}$ irata, agli adulteri amplessi di Parisina; alle buffonerie di un Barbiere di Siviglia, ai tradimenti, alle seduzioni, agli omicidî e simili. La religione, madre discreta, per mezzo de' sensi s' apre la via al cuore; seguendo, se così puó dirsi, l' uomo nelle case, nelle botteghe, nelle officine, sui campi, lo guida per vie molteplici al fine unico. Dominate dal suo spirito, tutte le opere umane divengono suoi strumenti, sono stimoli che potentemente a poggiar alto aiutano il sentimento; essa possiede la virtù di ca- campagna, e lo stesso solenne passaggio per la piazza, in oggi colla ciocca d'ulivo, e dopo il rendimento di grazie in duomo, ognuno va a pranzare a casa sua.

Cosi hanno termine le Rogazioni di Rovigno, codeste feste religioso-popolari, che l'uomo mettono in comunicazione con Dio e coi defunti, e coi fratelli I' accomunano e parte conservano delle antiche memorie e tradizione. C. Maria Caverza.

Oltre la piccola chiesa della Madonna delle Grazie, cui si visita ogni giomo, e il duomo, se ne visitano otto il primo di, undici il secondo e diecinove il terzo. Il territorio, sebben breve, altre ancora ne conta, e di più ne contava, come altresi la città, ora dirute.

\section{Saggio di dialetto Rovignese. (**)}

Al scuminscia a cantà cun gran bravuura:

Cun pano vjirdo, ruusso e culùr viula

Ningouna duona xi visteida ancuurs,

Nè cavj d'uoro indrjssa, ch'una sùla

Che cùl sù gran sprendùr la me 'namuura:

$\mathrm{E}$ tanto biella che meio nùn sè ùla

$\mathrm{X} e \mathrm{i}$, che gilla me ciùl Ia libarta, $\mathrm{E}$ la me fà dei e nóto suspeirà.

*) Abbiamo ereduto bene, nell impossibilità di dare coi tip i veri segni che determinino la pronunzia di molte parole del dialetto Revignese, d'innestare tutte quelle vocali contrassegnandole col corsivo che dovrebbero esser poste di sopra la susseguente, oppure omesse, ma che non restano mute esi pronunziano. - Inoltre locino che ai pronunziano eome fossero realmente due $a$ o dae $u$, dovrebbero avere una linoetla orizzontale; la dovebbero pur avere le vocal

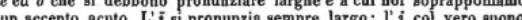
un accento acuto. $L^{\prime} i$ si pronunzia sempre largo; ${ }^{\prime} j$ col vero au

Fig. 1. La prima ottava del Saggio di dialetto Rovignese, con, sotto, le brevi annotazioni linguistiche (da "L'Istria”, a. I, 30 maggio 1846, n. 31-32, p. 127).

Radossi - A. Pauletich, op. cit., p. 258). Sonetti composero anche Antonio Angelini fu Stefano (1798-1863), e soprattutto Giuseppe Angelini (1762-1838) di cui sappiamo dal Glezer, come riportano i due autori (1976: 260), che fu "dottore, letterato, versato in poesia, avvocato, poi giudice". Egli scrisse, inoltre, le famose Sestine in difesa di Rovigno contro le critiche dell'abate Lazzaro Spallanzani. Ma alla poesia non fu estraneo neppure il padre di Piero Angelini, Giacomo dott. Angelini, che Radossi e Pauletich indicano come "il più insigne tra gli Angelini", e riportano dal Glezer: "Tutti i viventi lo ricordano per quanto fece di bene in generale al suo paese. Scrisse delle buone poesie, la maggior parte inedite" (op. cit., 264). Da quanto esposto, risulta evidente la difficoltà di attribuire a qualcuno di essi la composizione del Mendicante d'amore, tanto più che non si hanno finora conoscenze certe di loro componimenti in dialetto istrioto rovignese (eccetto per Piero Angelini, autore dei Lementi) ma solo in lingua italiana. Quindi, pur considerata la nota vocazione degli Angelini a dedicarsi alla composizione in versi, non possediamo, purtroppo, elementi certi per riportare a uno di loro la composizione del Mendicante. 
Ritornando all'analisi del poemetto, vediamo che esso rientra nella forma della poesia messa in musica, e quindi della poesia popolare che, come scrive Lorenzo Renzi, "è sempre accompagnata dalla musica: dalle villotte friulane ai mutos e mutettos sardi [...]" (Renzi 19914: 36), eseguita soprattutto nelle rappresentazioni giullaresche del XV secolo - e prima nella forma metrica di ottave di endecasillabi a rima ABABABCC ("ottava toscana") e nominata strambotto o rispetto, o ancora, come nel nostro caso, rispetto continuato. Era questo un particolare tipo di componimento in ottave in cui il discorso lirico, rusticale o amoroso o più spesso una contaminazione dei due, era appunto continuato entro una successione più o meno lunga di strofe di otto versi.

Tra le stanze per strambotti - come pure venivano chiamate - di argomento rusticale, vale la pena ricordare la Nencia da Barberino di Lorenzo de' Medici, cui Luigi Pulci rispose con la Beca da Dicomano, stravagante rappresentazione della già parodiata invocazione d'amore lorenziana. A prevalere, quindi, in questi componimenti d'intonazione popolareggiante ma di interpretazione aulica e che, a partire dal XV secolo prenderà la forma dell'ottava (Bontempelli 1978), era una ben dosata e felice commistione tra il sentimento d'amore, non scevro da punte di sensualità, a volte scopertamente scurrile, e una disposizione ridanciana, come è il caso della quinta ottava della Nencia:

"Ben si potrà tenere avventurato / Chi sia marito di sì bella moglie; / Ben si potrà tener in buon dì nato / Chi arà quel fioraliso senza foglie; / Ben si potrà tener santo e beato, / Che si contenti tutte le sue voglie / D'aver la Nencia e tenersela in braccio, / Morbida e bianca che pare un sugnaccio." (Bontempelli 1978: 251).

Da questa fonte citeremo d'ora in poi i versi tratti dal poemetto di Lorenzo de' Medici, pubblicato alle pagine 250-260. Va detto, però, che neppure Angelo Poliziano si mostrò ritroso verso questo modo di poetare. Anzi: la raffinata musicalità che troviamo nelle sue opere maggiori - in cui echeggiano motivi della poesia classica e trecentesca-, si riflette pure, con la stessa eleganza, nei rispetti continuati e spiccioli, in cui a predominare sono invece temi e immagini della vita e della poesia popolare fiorentina a lui coevi. E ciò, come rileva Bontempelli, perché "mentre il Magnifico e il Pulci tendono a mettere in burla la materia popolare, il Poliziano la tratta con lo stesso amore che l'erudita e mitologica" (Bontempelli 1978: 86). Si tratta difatti, nel caso di Lorenzo - escludendo da questa riflessione il Pulci - non di una adesione culturale o empatica al mondo rustico e subalterno che con i versi della Nencia andava rappresentando, quanto invece di una sua immedesimazione ironica e distaccata, mimetica, con quel mondo: un divertissement. Pur conoscendo bene quell'ambiente, Lorenzo è sempre consapevole dello iato intellettuale e di sensibilità che da quello lo divide. 
Infatti, come giustamente avverte Hermann Grosser: "L'autore della Nencia, pur rappresentando questo mondo primitivo e rustico, resta sempre il signore di Firenze e il raffinato letterato che è, e questo mondo lo interessa ma nel contempo lo diverte" (Guglielmino e Grosser 1996: 602). E sulla stessa scia del Grosser, osserva acutamente Franco Brevini come "la poesia in dialetto si è offerta sempre, non come esito naturale, ma come ribaltamento della poesia illustre, come antimodello di un modello che la precedeva e che le attribuiva senso" (Brevini 2010: 62).

Lo stesso carattere prevalentemente burlesco lo ritroviamo nel poemetto pubblicato dal Kandler sulle pagine de "L'Istria". Si tratta anche qui, come ha mostrato già esemplarmente Brevini per la Nencia lorenziana (Brevini 1999: 68), di una "struttura modulare", in cui l'autore sviluppa motivi derivanti dalla tradizione nenciale, distribuendoli poi, ordinatamente e quasi a mo' di entità autonome, entro il tessuto narrativo, avvicinandosi così alla tecnica del montaggio nella produzione cinematografica. A prevalere però nel Saggio kandleriano è il tono mesto, a tratti più apertamente lamentoso, mai però scoraggiato, del povero mendicante. Come a dire che, se pure nel poemetto del Vasgabrina vi è un'intenzione parodistica, questa non sconfina nell'esuberanza caricaturale che nella rappresentazione del mondo subalterno troviamo invece nei poemetti di Lorenzo o di Pulci; qui è maggiormente sentita, a tentare un accostamento con la tradizione, la presenza più pacata, quasi elegiaca, del Poliziano. Certo non vi mancano formulazioni topiche attraverso le quali l'autore prende le distanze dal suo personaggio, raffigurandolo entro una dimensione d'ingenua e popolaresca rozzezza, evidenziando così la disposizione ironica con cui si avvicina a questo mondo. È il caso, per esempio, degli ultimi due versi della quinta strofa dove, dopo un lungo elenco di privazioni e sofferenze causate dall'amore non corrisposto, tipiche di tale produzione, l'autore, non senza ironia, indirizza il discorso poetico dell'innamorato verso la "direttrice metaforica" (Jakobson 2010: 40): "Che par al grando amur i' jè sempro fan" ("Che per il grande amore ho sempre fame"), e metonimica: "Che sazià nu ma pol trj ster de pan" ("Che saziare non mi possono tre staia di pane"). Ma altri e vari sono ancora i richiami nel poemetto del Vasgabrina con la tradizione e con il topos del lamento: il motivo dello struggimento, della pena e della sofferenza causate dalla passione d'amore percorrono l'intero testo e trovano un illustre riferimento già nella Nencia del Magnifico. Scrive, infatti, il rovignese (I 5, 7, 8,): “[...] cùl su gran sprendùr la me "namuura: / [...] gjlla me ciùl la libartà, / E la me fa dei e nóto suspeirà" ("[...] col suo gran splendore m’innamora: / [...] lei mi toglie la libertà, / E giorno e notte mi fa sospirare"); e già Lorenzo (I 1, 2): "Ardo d'amore, e convienmi cantare / Per una dama che mi strugge il core". Troviamo poi nel rovignese il motivo della consunzione causata dal tormento amoroso $(\mathrm{V} 1,2)$ "La me và consumando douto quanto / 'Nchin 
che gninte de meio nùn restarò" ("Mi va consumando tutto quanto / Fino a che niente resterà di me") che ricalca, fedelmente, la stessa immagine del Vallera laurenziano (X 1-6): "La m'ha sì concio e in modo governato / Che più non posso maneggiar marrone, / Ed hammi drento così avviluppato / Ch'io non posso inghiottir già più boccone / E so' come un graticcio diventato". Non manca poi nel mendicante la descrizione - spesso in forma d'iperbole, e quindi non senza un ammiccante sorriso al lettore - delle bellezze della donna amata, sia fisiche sia morali, come pure l'elogio, vero e proprio panegirico, della sua condotta virtuosa e impeccabile (III 1-8):

Parchì xi tanta la sù gran baljssa
Che la fa 'namurà i piani e i monti,
A val piùn al sprendùr d'una su drjssa
Che duto 'l staato dei piùn grandi cónti;
I su custrumi bai, la su savjssa
Nun sa pol numerà cun duti i cuonti
Ningouna lengua dei nùn puodarávo
Le su vjrtù ch'al fià ghe mancaràvo. ${ }^{13}$

$E$ anche qui la vicinanza con i moduli espressivi e gli stilemi laurenziani è evidente. Ecco, infatti come Lorenzo fa vedere al pastore Vallera la sua Nencia (I 5): "Ella non trova di bellezza pare", o ancora - e qui riecheggiando parodicamente la lirica stilnovista, stilema da cui invece il rovignese si tiene sempre estraneo - (III 1-4): "Non vidi mai fanciulla tanto onesta / Né tanto saviamente rilevata: / Non vidi mai la più pulita testa, / Né sì lucente né sì ben quadrata".

Un altro elemento topico del genere è pure l'immagine - anche questa di antica tradizione - della pena d'amore quale causa, o eccesso, che porta allo sviamento, alla pazzia dell'amante giacché vittima del fatale sentimento. Nel Vasgabrina tale errore - che si risolve in impotenza e straniamento - viene efficacemente raffigurato con la metafora della prigione (VII 1-3): "Cumù i'nùn siè puoi duopo in sta parzon / Chi m'ebbio strassinà a patei cusseio, / Che dabuoto i'jè piersa la razon" ("Io non so poi come in questa prigione / Chi mi abbia trascinato a soffrire così, / Che di colpo ho peso la ragione"); passaggi, questi, che troviamo susseguentisi anche in Lorenzo. Si lamenta, infatti, Vallera (X 6-8, XI 1, 2): "Tanta pena mi dà e passïone; / Ed ho fatiche assai, e pur sopportole; / Ché m'ha legato con cento ritortole. // Io son sì pazzo della tua persona, / Che tutta notte io vo traendo guai". E ancora nei Rispetti continuati del Poliziano: "Io mi solevo andar libero e sciolto, /

13 "Perché tanta è la sua bellezza / Da far innamorare piani e monti, / Vale più lo splendore di una sua treccia / Che l'intera ricchezza dei più grandi signori; / I suoi bei costumi, la sua saggezza / Non vi è modo di enumerarli tutti / Nessuna lingua potrebbe dire / Le sue virtù ché il fiato gli mancherebbe." 
Or nelle forze sue mi tiene Amore. / [...] / Tu hai le redini in man del duro morso, / E di me puoi disporne al tuo comando" (Bontempelli 1978: 94).

Ma alla sofferenza e al vortice passionale dell'amante, antitetico è invece l'atteggiamento di fredda indifferenza e malcelata irrisione dell'amata nei confronti del giovane. Essa, insensibile e dura, ci riporta alla petrosa dantesca o al motivo dell' amante fatto oggetto di dileggio, come in Petrarca ([...] al popol tutto / favola fui gran tempo [...] $)^{14}$. Anche qui la crudeltà della donna non si ferma al rapporto interpersonale ma va oltre, fino a ricercare l'irrisione e la beffa anche del vicinato, come già lamentava Vallera, dove "molto si canzona" (XI 5) o, più esplicito: "Stato m'è detto che tu mi dileggi" (XIV 5). E il Vasgabrina, di riflesso (V 3-6): "E puoi ancuura la se dà gran vanto / Ch'incontra meio piùn doura la sarò; / E la vol malsipàme ancuura tanto / Ch'al mondo a le me'pale reidaro'" ("E poi ancora si dà gran vanto / Che contro me sarà ancor più dura; / E vuole farmi ancora tanto disperare ${ }^{15}$ / Che il mondo riderà alle mie spalle").

Abbiamo qui individuato alcuni parallelismi topici che rinviano a una vicinanza e a una lezione ben assimilata con gli esiti più notevoli della tradizione letteraria italiana - e poetica in particolare - da Dante ai grandi del Quattrocento. Ma altri se ne potrebbero elencare, accreditati tutti da quel particolare modus scribendi codificato dagli stilnovisti - e ancora prima dai lirici provenzali - e istituzionalizzato dalle rime del Petrarca. Un repertorio, questo, cui si continuerà incessantemente ad attingere, come ben dimostra anche il poemetto del Vasgabrina, immettendo sì delle varianti ma sostanzialmente giocando su stilemi, motivi, espressioni e immagini accolte e avvalorate dall'intera tradizione precedente. Così, tra gli altri calchi adottati dal Nostro, vi troviamo (VI 3, 4) il motivo tipico dei capelli biondi e gli "[...] uocci ciari / Ch' passa i córi de doute le bande" ("[...] occhi chiari / Che trapassano i cuori da parte a parte"), né manca l'efficacissima ma abusata figura dell'antitesi, qui però resa ancor più espressiva dalla disposizione chiastica (VIII 2): "I pjùro sempro noto e deì cantando" ("Piango sempre notte e dì cantando"), o l'invocazione - d'ariostesca memoria - dopo la sequela di lamenti, all' amata (IX 1-3, 8): “Ah! par pietà nùn stà scampame veia, / Nùn essi piùn cun meio cousseio spiataada, / Nùn me fa veivi piùn 'n malincuneia [...] / E ma fjsso butà 'n desparascion" ("Ah! per pietà non sfuggirmi, / Non essere più con me così spietata, / Non mi far vivere più in malinconia [...] / E farmi cadere nella disperazione"). Stilema, questo, molto frequente anche nei rispetti di Poliziano: "Per dio, madonna, donami soccorso, / Perch'io non mora giovinetto amando: / [...] / Soccorrimi oramai: merzè chiamando / Finir mi sento il core in te sperando" (Bontempelli 1978: 94).

\footnotetext{
${ }^{14}$ Voi ch'ascoltate in rime sparse il suono, vv. 9-10, in Petrarca (1964": 3).

${ }^{15}$ Per il verbo malsipame l'autore riporta nella nota: "Trattarmi male - farmi disperare".
} 
Il lamento del Vasgabrina si differenzia però dai componimenti simili per la mancanza - almeno per quanto interessa le strofe che ci sono pervenute - dell'elemento idillico della natura. Anzi, nei versi del nostro poeta è assente qualsiasi riferimento al mondo esterno, se non per una breve iperbole (VI 5-8): "Tante giùsse de aqua in douti i mari / Mei deigo che nùn xì, ne in bùsco giande, / Quanti suspeiri ch'jè tirà par teio" "'Tante gocce d'acqua in tutti i mari / Dico che non ci sono, né in bosco ghiande, / Quanti i sospiri che ho tratto per te"), ma anche qui esclusivamente in funzione del suo sentimento di afflizione, causato dall'innamoramento non corrisposto, tanto che "[...] scouro al zjil xì davantà par meio" ("[...] oscuro è diventato per me il cielo").

Ciò che invece avvicina il poeta de Il mendicante ai rispetti continuati del Poliziano (e ad alcune canzoni a ballo sia polizianee, sia laurenziane) èoltre a un sentimento più mite e l'assenza di eccessi iperbolici e caricaturali tipici dell'opera di Lorenzo e del Pulci - l'attenzione nei confronti della fugacità del tempo e della precarietà della vita umana e, in particolare, della caducità della sua età più bella: la giovinezza. Scrive, infatti, Vasgabrina $(\mathrm{X})$ :
Al diz: inchin ca zùvani i’ signémo
Almanco un può gudénse, parché puoi
Vieci sensa pensà i' daventarémo:
Né par nostro cuntento gnanca fjòi
De poudi cunsulasse i' nùn varémo:
De la muur donca femo 'l teibidòi,
Nùn lassemo passà sti ani bai,
Parchì s'i' passa i' nun' d' e turna mai. ${ }^{16}$

L'ottava del rovignese sembra qui un'efficace sintesi di consimili meditazioni sul veloce ed ineluttabile trascorrere del tempo. Meditazioni già elaborate, oltre che dal Poliziano, dal suo mecenate e compagno nella notissima Canzona di Bacco, entrambi modelli principali, possiamo arguire, cui si rifà il Vasgabrina. Ma mentre nel Poliziano il messaggio s'inserisce sempre entro un rapporto allocutivo ("Prendi bel tempo, innanzi che trapassi, / Gentil fanciulla, el fior degli anni tuoi”) (Bontempelli 1978: 89), e il tempo è inteso quasi esclusivamente come fattore deleterio e deturpante nei confronti della bellezza fisica della giovane ("Veggo cambiare el tuo vago sembiante, / La tua bellezza come un fior si fugge") (Bontempelli 1978: 88), nel Vasgabrina le coordinate ideologiche mutano sensibilmente, rivolgendosi il messaggio dell'autore non più alla donna esclusivamente

16 “Dice: fin che siamo giovani / Godiamo almeno un po', perché poi / Vecchi senza pensarci diventeremo: / Né conosceremo il diletto di avere dei figli / Con i quali poterci consolare: / Lasciamoci dunque prendere dall'amore, / Non lasciamo passare questi anni belli, / Perché se passano non ritorneranno più." 
bensì al noi, prefigurazione già squisitamente borghese della coppia unita nel vincolo matrimoniale e poi, soprattutto, familiare, con inoltre il preciso riferimento ai figli. Vincolo, questo, del tutto assente nei versi dei due quattrocentisti: sia in Lorenzo sia in Poliziano non troviamo accenno né al matrimonio né ai figli. Possiamo quindi rilevare come quest'ultimo elemento, a differenza degli altri fin qui riscontrati, allontani il Vasgabrina dai modelli, inserendolo invece pienamente nell'ideologia razionale e concreta del ceto borghese del suo tempo. Il Vasgabrina così innesta, sul modello della tradizione, il motivo moderno e previdenziale della famiglia e, inoltre, il godimento nel mendicante non è più visto in funzione esclusivamente edonistica come succedeva in Poliziano, in cui: “[...] '1 tempo vola, e non s'arreston l'ore / E la rosa sfiorita non s'appreza: / Dunque allo amante tuo fanne un presente: / Chi non fa quando può, tardi si pente" (Bontempelli 1978: 91), ma diventa bensì lo strumento per assicurarsi, da vieci, grazie ai figli, un previdenziale sostentamento.

Se a prevalere nel rispetto rovignese è quindi un tono di leggerezza amena $\mathrm{o}$, al più, di un'ironia moderatamente contenuta nella descrizione dello struggimento d'amore dell'amante, non vi manca però il compiacimento per il doppio senso e per il gusto dell'irrisione spicciola e sensuale, come nel caso della presentazione che l'autore fa della bella giovane subito dopo essersi soffermato sulle sue doti di virtù, saggezza e costumatezza. Il nome della giovane sembra così la cartina di tornasole, dietro la quale l'autore cela la vera immagine della ragazza. Essa si chiama
[...] la gran Gudenscia,
Che de majuure stade nùn de' xì
E biella purassè, de gran sapienscia.
Ma cateiva cùn meio ch'i' nùn puoi deì
Quanto la me fa pierdi la passenscia:
Se la me vuolta puoi le su palpjre
Le deliscie del zjil i’ vido vjre. ${ }^{17}$

Il nome è già un'esplicita rivelazione: esso, come un concentrato degli opposti, racchiude in sé il fiore della virtù "[...] biella purassè, de gran sapienscia" e l'asperità, la labilità (dal quinto all'ottavo verso: irascibile e capricciosa) - attributi tradizionalmente ascritti alla donna fino alle soglie dell'età moderna. Emerge così, dai versi del rovignese, una donna la cui femminilità cateiva trova esatto riscontro in quanto, a un secolo e mezzo di distanza dalla rappresentazione del Vasgabrina, scriverà della donna Nadia Fusini: "Effimera, volubile, qual piuma al vento, la donna è colei che non sa

17 “"...] la gran Godenzia, / Che di maggiori non ce ne sono state / Bella è assai, e di gran sapienza. / Ma cattiva con me ch'io non lo posso dire / Quanto mi fa perdere la pazienza: / Se poi mi rivolge le sue palpebre / Vedo le vere delizie del cielo". 
amare. Ma suscita amore" (Fusini 2004: 103). E, al di là dello struggimento del povero Andrja Uorgani, è lo scompiglio dell'autore reale ciò che ci si svela verso dopo verso dalla lettura del poemetto. Scompiglio che è provocato in lui da una figura femminile "dall'esistenza dispersa, frammentata, persa in un'evasione continua. Esistenza votata alla fuga" (Fusini 2004: 103), come fuggevole è, nella penultima ottava - l'unica in cui, possiamo dire, si riveli genuino l'afflato poetico - la bellissima e nitida immagine de " $i$ usài ch' $i$ ' zjra inturn'i neidi" ("gli uccelli che girano intorno ai nidi”), a rappresentare, con mirabile levità e non minore grazia, l'appressarsi a lei di "chi che [la] duura" ("chi [1'] adora"). Ma è soprattutto nei primi quattro versi che la poesia si scioglie dall'ingarbugliato e a volte sconnesso discorso poetico che aveva caratterizzato le precedenti stanze per farsi ora puro, spontaneo sentimento, confidenziale preludio all'aerea immagine di cui sopra:
Se biella ti me pari cù ti reidi
Biella ti son quando ti pjuri ancuura,
Cù ti trùvi baroufa, cù ti creidi
Chi ca ta vjdo douti se 'namuura,
Che cumù i usài ch' i' zjra inturn' i neidi
Cousseio "nturno ta ven chi che te duura;
Ningun se pol sazià ' $n$ le tu' baljsse.
Douti resta ligadi ' $n$ le tu' drjsse. ${ }^{18}$

E come suona dolce quest'incipit con valore concessivo! Attenua e smussa in un unico verso tutta l'aridezza e la convenzionalità di costrutti messi in rima e incasellati entro, a volte, una poco connessa - se non incoerente - o troppo artificiosa struttura logico-discorsiva. È il caso, per fare solo un esempio tra i molti che si potrebbero addurre, degli ultimi due versi della stanza 12: "Nùn ma n'inpuorta de cuuri a la muorto / Pur che cùn teio i'pudisso vi me'suorto" ("Non m'importa di correre alla morte / Purché con te potessi avere miglior sorte"). Qui invece sentiamo risuonare, semplice e lirico il ritmo della poesia. Innanzitutto nella cadenzata distribuzione degli accenti che determina, nell'intervallo speculare tra i dittonghi ie e ei, una fluidità espressiva, che si riflette nel parallelismo dei due primi versi e nell'antitesi fonico-semantica, quasi chiastica, reidi-pjuri. Un ulteriore effetto sonoro, poi, è dato ai versi dall'allitterazione della liquida $r$, compresa quasi sempre tra due vocali, per cui i versi si aprono a una melodica scorrevolezza che si protrae lungo l'intera ottava. Ma è soprattutto l'allitterazione $t$-ti che comparendo ben sedici volte in otto versi mette in forte risalto la

18 "Se bella tu mi appari quando ridi / Bella tu sei anche quando piangi, / Quando bisticci, quando gridi / Chiunque ti vede di te s'innamora, / Come gli uccelli che girano intorno ai nidi / Così girano intorno a te quelli che ti adorano; / Nessuno si può saziare delle tue bellezze. / Tutti restano avvinti nelle tue trecce". 
figura della donna desiderata, fin quasi a fondersi, a diventare un tutt'uno con i versi ${ }^{19}$. E il dettato poetico non scade in sofisma neanche quando, nei due ultimi versi della stanza, fa rimare baljsse (bellezze) con drjsse (trecce): di nuovo un parallelismo che si riflette nelle due uguali unità prosodiche delle due coppie di emistichi e nell'incipit antitetico "Ningun-Douti". E non sfugga, per ultimo, il motivo di boiardesca memoria - ricordiamo qui solo gli “inamorati augelli", i "vaghi augeleti” dal madrigale I dell' Amorum liber primus - (Boiardo 1927: 10) o, già prima, di trobadorica reminiscenza dove i versi in cui gli “"[...] aucel / Chanton, chascus enlor lati / Segon lo vers del novel chan" ("[...] uccelli / Cantano, ciascuno nella sua lingua / Secondo la melodia del nuovo canto") (Mölk 1986: 24), confermano quanto inizialmente accennato sull'esecuzione musicata di tali componimenti. Difatti, come scrive Beltrami, strambotto andrebbe riportato al provenzale estribot (Beltrami 1994), e da questo, quindi, al trobar, termine con il quale "si deve intendere non soltanto 'comporre poesie', ma anche 'comporre musica"" (Mölk 1986: 49); musica, quella del Mendicante rovignese, che, possiamo concludere, non dovette essere estranea all'alveo della tradizione cantata delle arie da nuoto e delle bitinàde rovignesi (Di Paoli Paulovich 2017).

\section{BIBLIOGRAFIA}

Beltrami, P. G. (1994). La metrica italiana. Bologna: Il Mulino.

Boiardo, M. M. (1927). Il Canzoniere. Torino: Unione tipografico-editrice torinese.

Bontempelli, M. (1978). Il Poliziano, il Magnifico, lirici del Quattrocento. Firenze: Sansoni.

Brevini, F. (1999). La poesia in dialetto. Tomo primo. Milano: Arnoldo Mondadori.

Brevini, F. (2010). La letteratura degli italiani. Milano: Feltrinelli.

Budicin, M. (2015). Da L'Istria del Kandler (1846-52) agli Atti XLV, Atti. Centro di Ricerche Storiche, Rovigno, vol. XLV, 7-56.

Cergna, S. (2012). La produzione poetica istriota dell'Istria sudoccidentale dal 1835 ad oggi. Tesi di dottorato. Zagreb: Filozofski fakultet u Zagrebu. Crevatin, F. (2013). Presentazione. In L. Benussi, Vocabolario italiano-rovignese e Appendice del vocabolario del dialetto di Rovigno d'Istria 1992-2013 (pp. 9-11). Rovigno: Comunità degli Italiani "Pino Budicin".

${ }^{19}$ Anche qui, come nella lettura che della poesia di Leopardi $A$ Silvia ha dato Stefano Agosti, la disseminazione nel testo della consonante $t$ potrebbe forse evocare il vocativo $t u$, il pronome atono $t i$ e l'aggettivo $t u o$, rendendo così palese l'occultamento della "libido vocativa" che si celerebbe dietro l'insistente richiamo della donna. Cfr. Renzi (1991: 25-26). 
Di Paoli Paulovich, D. (2017). La musicalissima Rovigno nei suoi generi di canto. Atti. Centro di Ricerche Storiche, Rovigno, vol. XLVII, 439-493. Fusini, N. (2004). La passione del male. In E. Pellegrini, Le spietate. Cava de' Tirreni: Avagliano Editore.

Guglielmino, S. e Grosser, H. (1996). Il sistema letterario. Quattrocento e Cinquecento. Milano: Principato.

Jacobson, R. (20104). Saggi di linguistica generale. Milano: Feltrinelli.

"L'Istria", a. I, 28 marzo 1846, n. 16-17.

"L'Istria", a. I, 11 aprile 1846, n. 19-20.

"L'Istria", a. I, 30 maggio 1846, n. 31-32.

Mölk, U. (1986). La lirica dei trovatori. Bologna: Il Mulino.

Petrarca, F. (19644). Canzoniere. Torino: Einaudi.

Radossi, G. e Pauletich, A. (1976). Compendio di alcune cronache di Rovigno di Antonio Angelini. Atti VI, I, 246-374). Rovigno: Centro di ricerche storiche di Rovigno.

Radossi, G. (2014). Il carteggio Pietro Kandler - Tomaso Luciani (18431871). Rovigno: Centro di ricerche storiche di Rovigno.

Renzi, L. (19914). Come leggere la poesia. Bologna: Il Mulino.

Salimbeni, F. (1991). Pietro Kandler. In F. Semi, Istria e Dalmazia. Uomini e tempi (Vol. I, pp. 295-297. Trieste: Del Bianco Editore.

Tekavčić, P. (1980). Il posto dell' istroromanzo nella Romània Circumadriatica. SRAZ XXIV, 21-46.

Ursini, F. (1989). Istroromanzo. Storia linguistica interna. In G. Holtus et al. (a cura di), Lexikon der Romanistischen Linguistik (pp. 537-548). Tübingen: Max Niemeyer Verlag.

\section{A CONTRIBUTION TO THE ISTRIOT DIALECT OF ROVIGNO: OBSERVATIONS ON THE ESSAY IN THE ROVIGNO DIALECT- THE BEGGAR OF LOVE BY ANDRJA UORGANI \\ (EDITED BY PIETRO KANDLER)}

\section{Summary}

The paper presents a comparative stylistic and metric analysis between a poem in the Istriot dialect of Rovigno, published in 1846, and texts by poets of the classical Italian literary style of the fourteenth century. The comparison reveals the typical style and motifs of courtly poetry, such as unrequited love, the suffering caused by it, the teasing of the lover by the frivolous young girl, but also the ironic - and barely hidden - expression of the author, all presented popularly through the intelligent use of the Istriot dialect of Rovigno.

Keywords: Rovigno, Dialect, Istriot, poetry, Kandler, Istria. 